\title{
Pre-main sequence multiple systems
}

\author{
Hervé Bouy ${ }^{1}$ \\ ${ }^{1}$ Centro de Astrobiologia, INTA-CSIC, P.O. Box - Apdo. de correos 78, Villanueva de la \\ Cañada Madrid 28691, Spain \\ email: hbouy@cab.inta-csic.es
}

\begin{abstract}
It is now well established that the majority of young stars are found in multiple systems, so that any theory of stellar formation must account for their existence and properties. Studying the properties of multiple star systems therefore represents a very powerful approach to place observational constraints on star formation theories. Additionally, multiple systems offer other advantages. They provide the most accurate and unambiguous way to measure masses, using orbital fitting and Kepler's laws, and even the stellar radius in the special case of eclipsing binaries. They also allow to compare the properties of 2 coeval objects with different masses, providing important tests for the evolutionary models.
\end{abstract}

Keywords. Stars: binaries: general, Stars: formation

\section{Introduction: Parameters of interest}

Multiple systems are very diverse: binaries can be wide or tight, the 2 components can have very different masses, and we even know triple, quadruple and higher order systems. It would be impossible to review all the different properties in just $15 \mathrm{~min}$, and this presentation will focus on three major parameters: the multiplicity frequency, the distribution of mass ratio and of separation.

These various properties depend directly on the mechanisms at work during the early stages of stellar formation and in the subsequent few million years. Early studies of multiplicity focused essentially on the frequency of multiple systems, but the advent of powerful and sensitive instruments allowed astronomers to investigate in more details the statistical properties of multiple systems and in particular their distributions of mass ratio and separation. These properties, and how they depend on the age, the environment and the mass, can in turn be readily compared to theoretical predictions and numerical simulation outputs.

\section{Multiplicity vs Environment}

Early studies of multiplicity have found a significantly higher rate of multiple systems in young loose associations than in young clusters. Young clusters on the other hand seem to have multiplicity properties similar to those observed for the nearby field population. Early numerical simulations showed that a clustered population can indeed evolve into the field binary distribution through dynamical decay, providing additional evidence that the field population most likely formed in a clustered environment rather than in loose associations.

\section{Multiplicity vs Primary's mass}

Figure 1 shows the observed binary fraction as a function of spectral type. The results plotted in this figure come from studies with very different levels of sensitivity and 

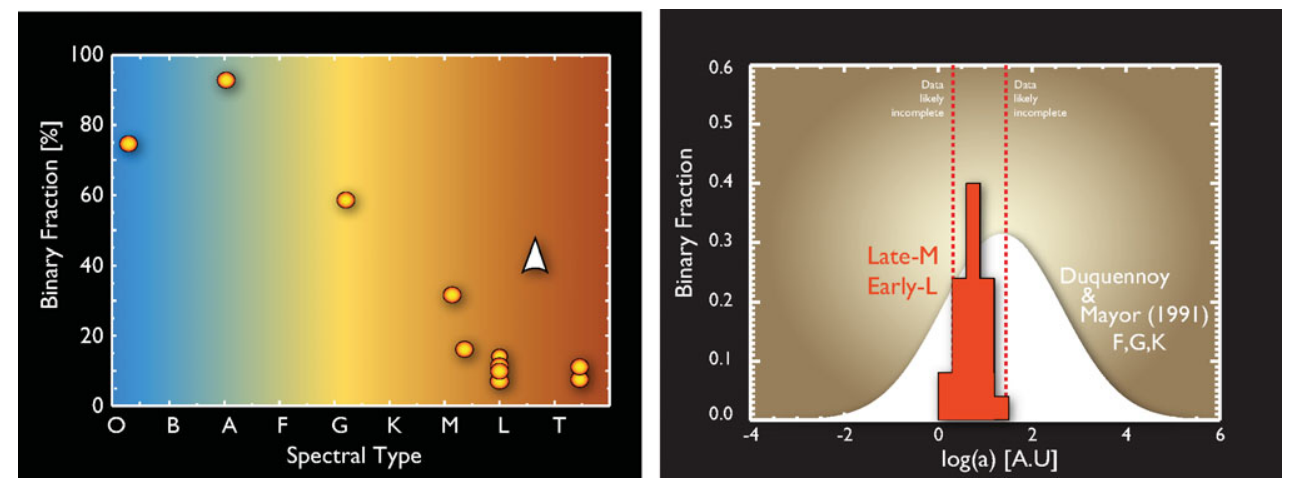

Figure 1. Left Panel: Multiplicity frequency as a function of Spectral Type. Values from Maíz Apellániz (2010), Mason al. (2009), Tokovinin \& Smekhov (2002), Duquennoy \& Mayor (1991), Siegler et al. (2005), Leinert \& al. (1997), Fisher \& Marcy (1992), Reid \& Gizis (1997), Goldman et al. (2008), Burgasser et al. (2003), Joergens (2006), Bouy et al. (2003, 2006), Maxted \& Jeffries (2005),Basri \& Reiners (2006). Right Panel: Distribution of separation for solar mass stars from Duquennoy \& Mayor (1991) and very low mass stars Bouy et al. (2003).

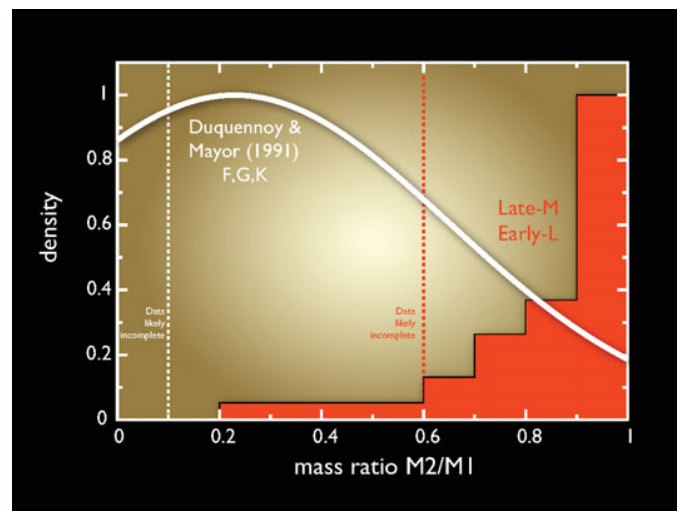

Figure 2. Distribution of mass ratio for field solar mass stars from Duquennoy \& Mayor (1991) and very low mass stars Bouy et al. (2003).

completeness. For example the measurements obtained for the ultracool objects are missing the closest binaries, and the real binary fraction could add up to 40 or $50 \%$. The value reported at the high mass end is also an upper limit, and the binary fraction is expected to be nearly $100 \%$. A direct comparison is therefore strictly not possible, but this figure nevertheless displays a clear trend of decreasing multiplicity fraction with the mass of the primary.

Figure 1 shows the distribution of separation for solar type stars and very low mass stars and brown dwarfs. The two distributions are gaussian, the very low mass star distribution being a scaled down version of the solar mass one. The much narrower separation range covered to date for very low mass objects prevents a more detailed quantitative comparison.

Finally, although the current surveys for very low mass stars were not sensitive to small mass ratio, the mass ratio distributions for solar type stars and very low mass stars are significantly different, the later one displaying a clear preference for equal mass systems as illustrated int figure 2 . 

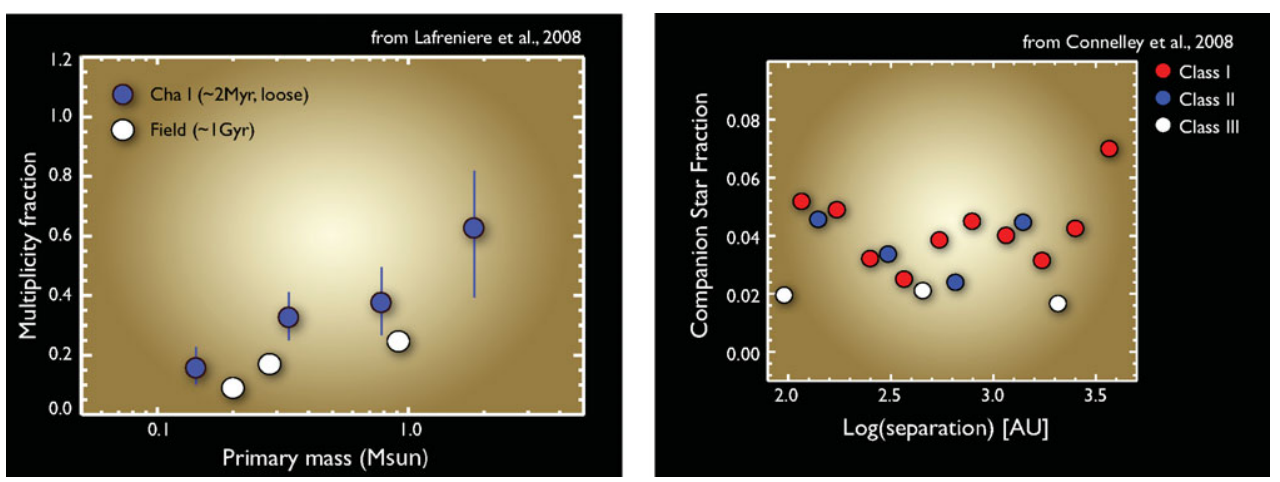

Figure 3. Left Panel: Multiplicity frequency as a function of mass for Cha I members and field solar mass stars. Figure from Lafreniere et al. (2008). Right Panel: Distribution of separation for class I, II and III. Figure from Connelley et al. (2008).

\section{Multiplicity vs Age}

Most of the multiplicity properties are set in the very early stages of star formation. Dynamical decay plays a very active role in the first few million years. Figure 3 shows the multiplicity frequency of the young loose association Chameleon I compared to that of the solar neighborhood as measured by Lafreniere et al. (2008). As explained earlier, the young association has a multiplicity frequency about twice higher than that of field stars, and the multiplicity frequency decreases with decreasing mass. In such a lose association of a few million years, dynamical interactions are unlikely to modify further the multiplicity rate. In a denser cluster, gravitational interactions would on the contrary be more frequent and modify the multiplicity frequency until it becomes similar to that of field stars. We therefore see that the dependence on the age is in fact tightly related to the initial conditions and environment.

Figure 3 shows the distribution of separation of class I, class II and class III sources as reported in Connelley et al. (2008). All three are relatively flat over the small separation range covered here and within the uncertainties, and once again this figure illustrates the higher multiplicity frequency among young objects members of loose associations.

The dependence on the age of the distribution of mass ratio has not been studied in great details, mostly because observations of deeply embedded protostars or young stellar objects are challenging with the current instrumentation. The best results have been obtained for high mass stars, which are relatively brighter and easier to observe. At young ages, the distribution of mass ratio for high mass star companions seem to follow random sampling, while at older evolutionary stages, the least massive companions have been ejected and the mass ratio distribution peaks towards unity.

\section{Comparing observations to simulations}

Since the aim of this conference is to discuss about computational star formation, and therefore to put together observations, theories and numerical simulations, a few words of cautions for both observers and theoreticians should be given.

Interpreting the observations can be a perilous exercise. All the results mentioned in this presentation were obtained within given instrumental limitations. For example, direct imaging using adaptive optics provides similar spatial resolution than speckle interferometry but different limits of sensitivity. Radial velocity surveys are so far limited to relatively bright sources, and often miss the long period companions. None of these 
surveys cover the entire separation range and mass ratio range of binary populations. As such, differences in measured binary frequencies could be the result of differing separation or mass ratio distributions. General trends can be seen, but more complete observations are required to make quantitative and definitive comparisons. For all these reasons, one must be very careful when comparing observational results to theoretical or numerical predictions. Any parameter must be compared strictly within the specific range covered by the observations, and any article presenting observational results should describe with great details the specific domain covered by the observations.

\section{Conclusions and perspectives}

Even though the current observational and numerical limitations prevent a detailed and quantitative comparison of observations to numerical predictions, tremendous progresses have been made over the past decade regarding the multiplicity properties of pre-main sequence stars. These have brought crucial informations and shed a new light on our understanding of stellar formation. The development of new instrumentation for both high resolution imaging, high spectral resolution, high astrometric accuracy, as well as the advent of new multi-epoch surveys, give very exiting perspective for this field of research. In this short presentation, I gave only a very shallow overview of the multiplicity properties. Multiple systems are very rich, and many more of their properties are extremely important. The distribution of eccentricity, the properties of their discs, the occurrence of high order multiple systems, and the presence and properties of planets in multiple systems (as we know multiple systems hosting planets) provide very exiting perspectives for the study of stellar formation.

\section{References}

Basri, G. \& Reiners, A. 2006, AJ, 132, 663

Bouy, H., Brandner, W., Martín, E. L., Delfosse, X., Allard, F., \& Basri, G. 2003, AJ, 126, 1526

Bouy, H., Martín, E. L., Brandner, W., Zapatero-Osorio, M. R., Béjar, V. J. S., Schirmer, M., Huélamo, N., \& Ghez, A. M. 2006, A\&A A, 451, 177

Burgasser, Adam J., Kirkpatrick, J. Davy, Reid, I. Neill, Brown, Michael E., Miskey, Cherie L., Gizis, \& John E. 2003, ApJ, 586, 512

Connelley, Michael S., Reipurth, Bo, Tokunaga, \& Alan T. 2008, AJ, 135, 2526

Duquennoy, A. \& Mayor, M. 1991, A\&A, 248, 485

Fisher D. \& Marcy G. 1992, ApJ, 396, 178

Goldman, B., Bouy, H., Zapatero Osorio, M. R., Stumpf, M. B., Brandner, W., Henning, T. 2008, A\&SA, 490, 763

Joergens, V. 2006, $A \mathscr{E} A$, 448, 655

Lafrenière, David, Jayawardhana, Ray, Brandeker, Alexis, Ahmic, Mirza, van Kerkwijk, \& Marten H. 2008, ApJ, 643, 844

Leinert, C., Henry, T., Glindemann, A., \& McCarthy, D. W., Jr. 1997, AJ, 325, 159

Maíz Apellániz, J. 2010, A\&SA, arXiv:1004.5045

Mason, Brian D., Hartkopf, William I., Gies, Douglas R., Henry, Todd J.; Helsel, \& John W. $2009, A J, 137,3358$

Maxted, P. F. L. \& Jeffries, R. D. 2005, MNRAS, 362, 45

Reid, I. N. \& Gizis, J. 1997, AJ, 114, 1992

Siegler, Nick, Close, Laird M., Cruz, Kelle L., Martín, Eduardo L., Reid, \& I. Neill 2005, ApJ, 621, 1023

Tokovinin, A. A. \& Smekhov, M. G 2002, A\& A, 382, 118 